\title{
Preparation of Aragonite Whiskers by Dolomite
}

\author{
W.R Xu $\mathrm{u}^{\mathrm{a}}, \mathrm{J}$ Chen and Z.L Huang \\ School of Materials and Engineering, Wuhan Institute of Technology, Wuhan 430074, China
}

\begin{abstract}
Aragonite $\mathrm{CaCO}_{3}$ with complicated morphologies needle-like structrues have been successfully synthesized through dolomite as raw materials by the decomposition of urea. The phase of the aragonite whiskers were characterized by X-ray diffraction and Fourier transform infrared, the structrue of the samples was characterized by scanning electron microscope and the effects of different ratio of materials were studied. The result show that the concentration of urea was found to have an effect on the morphology of aragonite crystals. All the prepared crystals were almost aragonite and there was no phase under varied experiment conditions.
\end{abstract}

\section{Introduction}

Aragonite whisker is a new kinds of calcium carbonate material under development recently. It is a mineral of common occurrence with very high mechanical strength[1]. The aragonite crystals were initially separated from the hot solution saturated with carbon dioxide, or by addition of calcium chloride to a hot solution of ammonium or sodium carbonate, when calcium carbonate crystallized. Water-soluble additives, such as, polymers[2], inorganic metal ions[3], and surfactants[4], have been widely used to modify the sharps and control the nucleation and growth of aragonite crystals. In order to synthesize aragonite whiskers, generally, higher temperature, low concentration of salt carbonate solution were to the benefit of the formation of aragonite whiskers[5].

Typically, the uniform needle-like aragonite whiskers were obtained by aging solution of calcium salts with the presence of urea at $90^{\circ} \mathrm{C}$ by L Wang. While Yu and co-workers synthesized pure aragonite crystals with rod-like shape in aqueous solution of 1:3 ratio of urea to water, and needle-like crystals made of aragonite nanorods with 1:1 ratio of urea to water without any organic additives[6].

In numerous studies, calcium carbonate whiskers are a new kind of inorganic single crystal with a production cost of about $\$ 236$ per ton. These crystals have been used as microscopic reinforcements in cementitious composites to improve toughness and reduce shrinkage. With a length of 20-30 um and a diameter of 0.5-2 um, $\mathrm{CaCO}_{3}$ whikers differ from other widely used fibers, like steel, glass, or polyvinyl alcohol fibers, in terms of the "particle-like" small size and large specific surface area. Compared with the addition of steel, glass, or PVA macrofibers, the same content of $\mathrm{CaCO}_{3}$ whiskers added in cementitious composites may cause fresh pastes[7].

\footnotetext{
a W.R Xu:740146159@qq.com
} 


\section{Experimental}

\subsection{Material}

All starting materials, dolomite(provided by LingShou HengDa minerals processing factory in Hebei, China), hydrochloric acid(obtained from XiLong chemical CO,. LTD. about 37\% of mass fraction), urea $\left(\mathrm{NH}_{2}\right)_{2} \mathrm{CO}$, obtained from XiLong chemical CO, LTD.) were of A.R. Grade. The water used in this experiment was deionized water.

\subsection{Synthesis procedure}

$6.0 \mathrm{~g}$ dolomite was mixed with $25 \mathrm{ml}$ hydeochiloric acid, in the presence of urea in $90^{\circ} \mathrm{C}$ bath as described. Specifically, equal volumes of urea solution were firstly at room temperature. Then transparent solution was transferred into a $60 \mathrm{ml}$ reaction still. Then, the reaction still was incubated and unstirred in a $90^{\circ} \mathrm{C}$ water bath for $24 \mathrm{~h}$. All of the particle nucleated and grew inside the reaction still, not on the wall of the flask. The precipitate was finally dried in a vacuum oven overnight at $100^{\circ} \mathrm{C}$. The synthesized condition of the aragonite whiskers was listed as in Table 1 . The concentration of dolomite was kept at 1.0M, and the concentration of urea was varied from 1.0 to $2.0 \mathrm{M}$.

\subsection{Characterization of the particles}

The phase composition and structure were identified by $\mathrm{X}$-ray diffraction recorded on a(Bruker $\mathrm{XD}-5 \mathrm{~A}) \mathrm{X}$-ray powder diffractometer with $\mathrm{Cu} \mathrm{K} \alpha$ radiation $(\lambda=1.5405 \AA)$ at scanning rate of $4^{\circ} \mathrm{min}^{-1}$. The infrared spectra were characterizated by using Bruker Fourier transform infrared (FT-IR) spectrometer on $\mathrm{KBr}$ pellets. The morphology and structure of as-prepared particles were examined by JEM-2210LV scanning electron microscope operating at an accelerating voltage of $20 \mathrm{kV}$ after sputtered gold film. The average grain size D was determined from Scherrer`s equation:

$$
\mathrm{D}=\mathrm{K} \lambda / \beta \cos \theta
$$

In the equation(1), $\mathrm{K}$ is expressed as dimensionless constant, $\lambda$ is expressed as wavelength of the $\mathrm{X}$-ray radiation $(1.5405 \AA), \beta$ is the full width at half-maximum of the diffraction peak, and $\theta$ is half of the diffraction angle.

\section{Result and discussion}

\subsection{XRD analysis}




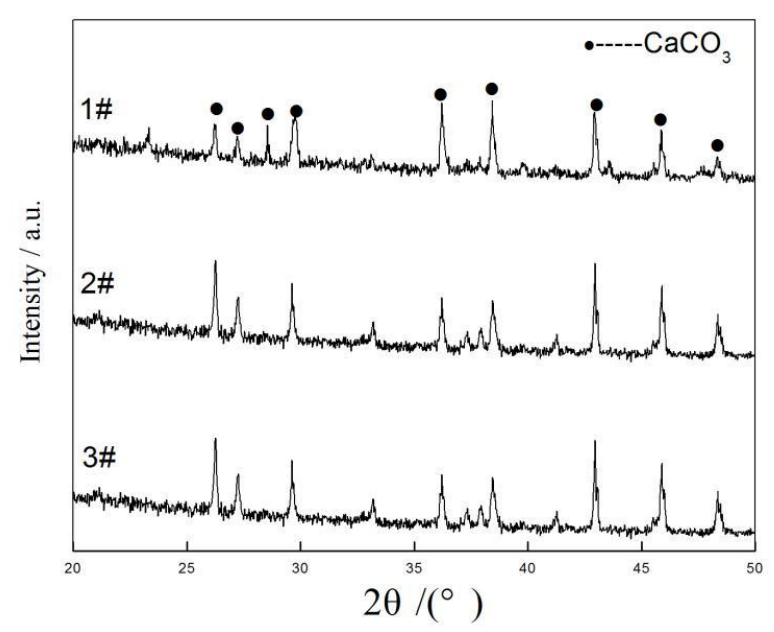

Figure.1 The XRD patterns of samples(1\#, 2\#,3\#)

The above structures were showed by the X-ray diffraction patterns in Figure. 1. All of the samples of the diffraction peaks, compared with the standard JCPDS files (aragonite: 41-1475; calcite: 05-0586; calcite,magnesian: 43-0697), in the XRD patterns clearly showed the peaks corresponding to $\mathrm{h} \mathrm{k} 1$ of $111,021,012,200,112,130,122$ et al., which agreed with those of aragonite phase. No other polymorph of $\mathrm{CaCO}_{3}$ was detected in the XRD pattern. The sharp and strong peaks indicated that $\mathrm{CaCO}_{3}$ was well crystalline. Scherrer's equation was applied to (lllll $\left.\begin{array}{lll}1 & 1 & 1\end{array}\right)\left(\begin{array}{lll}0 & 2 & 1\end{array}\right)\left(\begin{array}{lll}2 & 2 & 1\end{array}\right)$ peaks and provided a mean grain size D shows that the average crystalline size for the prepared $\mathrm{CaCO}_{3}$ crystals around $35.6-38.7 \mathrm{~nm}$, indicating that the resulted aragonite clusters were composed of aggregates of crystals.

\subsection{SEM analysis}

The scanning electron microscopy images of the fabricated $\mathrm{CaCO}_{3}$ crystals with $1 \mathrm{M}$ of dolomite, in varied concentration of urea were shown as in Fig.2. All pictures in Fig.2 showed that the prepared $\mathrm{CaCO}_{3}$ particles exhibited mostly needle-like. When the concentration of urea was $1.0 \mathrm{M}$, the needle-like aragonite with about 15 16 of aspect ratio, which was defined as the ratio of length to diameter, was obtained as Fig.2(1\#), and from which some of the crystals showed the tendency of orientation and needle-like structure. Fig.2(2\#) exhibits majority of aragonite crystals with clavate needle-like structure, made of the rods approximately with 9 10 of aspect ratio in addition to minority of needle-like crystals, the bundle-like crystals were formed. with $1.5 \mathrm{M}$ concentration of urea. With the concentration of urea increased to $2 \mathrm{M}$, the lager needle-like crystals were observed in Fig.2(3\#) with about $6 \sim 7$ of aspect ratio, the bundle-like crystals and dumbbell-like crystals were observed, the aggregation phenomenon of whiskers were also observed. 


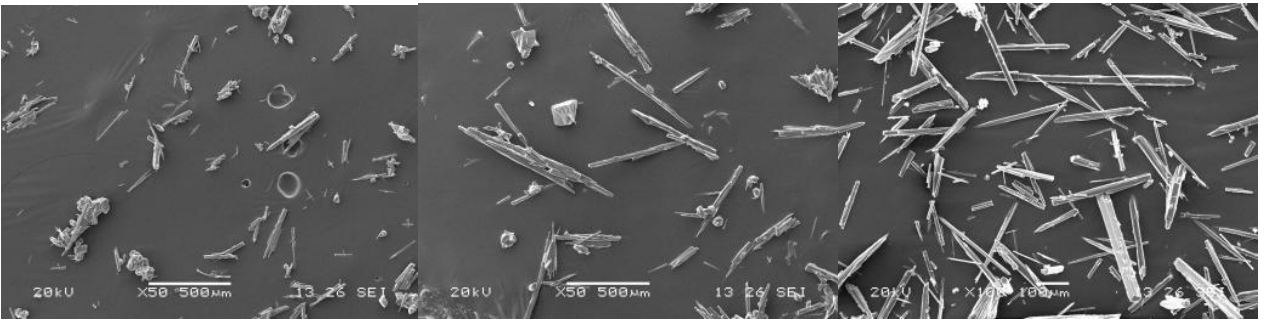

Figure.2 SEM images of aragnite crystals preparad at varid concentration of urea(1\#, 2\#, 3\#)

\subsection{FT-IR analysis}

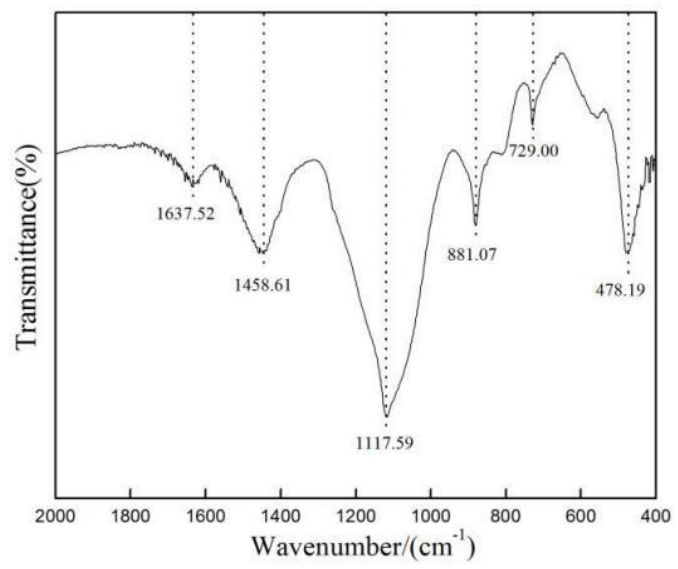

Figure.3 FT-IR spectrum of aragonite(1\#)

The aragonite phase of the sample was further confirmed by Fourier transform infrared spectroscopy spectrum shown in Figure.3. All spectrum spectra in Figure.3 showed vibrational bonds at $1117.59 \mathrm{~cm}^{-1}$ that can be attributed to the characteristic aragonite symmetric carbonate stretching $\left(v_{1}\right.$ mode). The spctrum spectra sharp peak located at $729.00 \mathrm{~cm}^{-1}$ was associated with the carbonate in-plane bending modes $\left(v_{4}\right)$ of aragonite. Moreover, the bands at about $881.07 \mathrm{~cm}^{-1}$ can also be attributed to the out-of-plane bending $\left(v_{2}\right.$ mode) vibration. For aragonite, the positions of the corresponding $v_{2}$ vibrations appear at about $875 \mathrm{~cm}^{-1}$, the vibration appears at $881.07 \mathrm{~cm}^{-1}$, is very similar and confirmed as the pure phase of aragonite. The two characteristic absorption peaks labled by number, located at $1637.52 \mathrm{~cm}^{-1}$ and $1458.61 \mathrm{~cm}^{-1}$, were assigned to the $\mathrm{C}=\mathrm{O}$ stretching vibration and $\mathrm{C}-\mathrm{O}$ bending vibration.

\section{4 conclusion}

In summary, aragonite $\mathrm{CaCO}_{3}$ with complicated morphologies needle-like structrues have been successfully synthesized through dolomite as raw materials by the decomposition of urea. The experiment parameters, such as the concentration of urea was found to have an effect on the morphology of aragonite crystals. All the prepared crystals were almost aragonite and there was no phase under varied experiment conditions. The reduction of aspect ratio of aragonite whisker was focued with increasing of concentration of urea under invariant conditions of dolomite. 


\section{References}

1. A.L. Litvin, S.Valiyaeettil, D.L. Kaplan, Template-directed synthesis of aragonite under supramolecular hydrogen-bonded Langmuir monolayers, Adv. Mater. 9, 124(1997).

2. Y. Pan, X. Zhao, Y. Sheng et al, Biomimetic synthesis of dendrite-shaped aragonite particles with single-crystal feature by polyacrylic acid, Colloids Surf. A: Physicochem. Eng. Asp. 297(2007).

3. J. Yu, M. Lei, B. Cheng, X. Zhao, Facile preparation of calcium carbonate particles with unusual morphologies by precipitation reaction, J. Cryst. Growth 261, 566(2004).

4. Z. Nan, X. Chen, Q. Yang, Structure transition from aragonite to vaterite and calcite by the assistance of SDBS, J. Colloid Interface Sci. 325(2008).

5. Z. Hu, Y. Deng, Synthesis of needle-like aragonite from calcium chloride and sparingly soluble magnesium carbonate, Powder Technol. 140, 10(2004).

6. S. Chen, S. Yu, J. Jiang, Polymorph discrimination of $\mathrm{CaCO}_{3}$ mineral in an ethanol/water solution: formation of complex vaterite superstructures and aragonite rods, Chen. Mater. 18, 115(2006).

7. M. Cao, C. Zhang, Mechanical response and shrinkage performance of cementitious composites with a new fiber hybridization. Constr Build Master. 57, 45(2014). 